\title{
Fully Integrated, Low Drop-Out Linear Voltage Regulator in 180 nm CMOS
}

\author{
Yosef-Hay, Yoni; Larsen, Dennis Øland; Llimos Muntal, Pere; Jørgensen, Ivan Harald Holger
}

Published in:

Analog Integrated Circuits and Signal Processing

Link to article, DOI:

$10.1007 / \mathrm{s} 10470-017-1012-5$

Publication date:

2017

Document Version

Peer reviewed version

Link back to DTU Orbit

Citation (APA):

Yosef-Hay, Y., Larsen, D. Ø., Llimos Muntal, P., \& Jørgensen, I. H. H. (2017). Fully Integrated, Low Drop-Out Linear Voltage Regulator in $180 \mathrm{~nm}$ CMOS. Analog Integrated Circuits and Signal Processing, 92(3), $427-436$. https://doi.org/10.1007/s10470-017-1012-5

\section{General rights}

Copyright and moral rights for the publications made accessible in the public portal are retained by the authors and/or other copyright owners and it is a condition of accessing publications that users recognise and abide by the legal requirements associated with these rights.

- Users may download and print one copy of any publication from the public portal for the purpose of private study or research.

- You may not further distribute the material or use it for any profit-making activity or commercial gain

- You may freely distribute the URL identifying the publication in the public portal 


\title{
Fully Integrated, Low Drop-Out Linear Voltage Regulator in $180 \mathrm{~nm}$ CMOS
}

\author{
Yoni Yosef-Hay • Dennis Øland \\ Larsen • Pere Llimós Muntal • Ivan \\ H.H. Jørgensen
}

Received: date / Accepted: date

\begin{abstract}
This paper presents a capacitor-free low dropout (LDO) linear regulator based on a dual loop topology. The regulator utilizes two feedback loops to satisfy the challenges of hearing aid devices, which include fast transient performance and small voltage spikes under rapid load-current changes. The proposed design works without the need of a decoupling capacitor connected at the output and operates with a $0-100 \mathrm{pF}$ capacitive load. The design has been taped out in a $0.18 \mu \mathrm{m}$ CMOS process. The proposed regulator has a low component count, area of $0.012 \mathrm{~mm}^{2}$ and is suitable for system-on-chip integration. It regulates the output voltage at $0.9 \mathrm{~V}$ from a $1.0 \mathrm{~V}-1.4 \mathrm{~V}$ supply. The measured results for a current step load from $250-500 \mu \mathrm{A}$ with a rise and fall time of $1.5 \mu \mathrm{s}$ are an overshoot of $26 \mathrm{mV}$ and undershoot of $26 \mathrm{mV}$ with a settling time of $3.5 \mu$ s when $C_{L}$ between $0-100 \mathrm{pF}$. The proposed LDO regulator consumes a quiescent current of only $10.5 \mu \mathrm{A}$. The design is suitable for application with a current step edge time of $1 \mathrm{~ns}$ while maintaining $\Delta V_{\text {out }}$ of $64 \mathrm{mV}$.
\end{abstract}

Keywords Linear Voltage Regulators • Low Drop-Out • Capacitor-free • Capacitor-less · Dual-Loop · Fast Transient Response

\section{Introduction}

Nowadays, linear voltage regulators are important components integrated circuits. For on chip power management, where multiple supply voltages are used, low drop-out (LDO) voltage regulators play an important role. Improving power management will help to extend the battery life and could increase the

Yoni Yosef-Hay

Department of Electrical Engineering, Electronics Group, Technical University of Denmark (DTU), Building 325, Kgs., 2800 Lyngby, Denmark

E-mail: : s154607@student.dtu.dk 
use of portable devices. As the industry is pushing towards complete systemon-chip (SoC) design solutions, including improving power management, LDO voltage regulators becoming a main factor. They are needed to provide voltage supplies for circuits with steep load transients and for protecting sensitive circuits from supply voltage disturbances. Their superior noise-ripple rejection over switching regulator makes them suitable to supply noise sensitive circuits. Linear regulators have some advantages over switch mode power supplies as they provide lower output noise, less electromagnetic emission, high power supply rejection ratio (PSRR) and are easy to integrate on-chip within a small area while maintaining an accurate output voltage.

In portable devices such as hearing aids, there is a strict requirement on area. The number of discrete components must be minimized, as the electronics must fit in the ear canal. Implementing a capacitor free LDO regulator will help to reduce the overall size by eliminating the large output decoupling capacitor and increase the reliability of the system. On the other hand, for a voltage regulator without an on-chip capacitor (usually referred as capacitorfree or capacitor-less) does not have a large capacitor to set the dominant pole and stable the circuit. In the application at hand the estimated capacitance of load circuitry is between $0-100 \mathrm{pF}$. The absence of an output capacitor rises issues in the transient response, $\Delta V_{\text {out }}$ (undershoot and overshoot) that will be larger and there will be an increase of the settling time. Moreover a large output capacitor ensures stability as it will set the dominant pole and acts as a supply for the frequency components of the current load, $I_{L}$, outside the bandwidth of the regulator.

Removing the external capacitor requires the circuit to overcome the transient response and stability issues mentioned. There have been a number of capacitor-free topologies suggested in the literature. This previous research mainly focus on improving the transient performance [1] - [4]. One approach is to use active feedback and slew-rate enhancement circuit [5]. Another approach is a LDO structure with a three-stage amplifier and damping-factorcontrol frequency compensation [1] or utilizing voltage spike detection [3]. Articles [13], [14] suggested an adaptive biasing technique, to increase the bias current according to the magnitude of $I_{L}$. All these approaches and others ( [11] and [12] ) result in a rather complex design, large area and normally high quiescent current.

Some voltage regulators use NMOS as pass device [15]. These designs can be smaller in size due to the higher charge carrier mobility in NMOS devices, thus enabling the same drain current with a smaller area. A PMOS pass element reduce the minimum required voltage drop across it. The advantage of using PMOS as pass transistor is that the supply voltage does not need to be significantly higher than the output voltage. Smaller voltage headroom results in less power dissipation, essential for devices like hearing aids.

Modern hearing aids need to fit in the ear canal, no integrated chip intended for such applications can be larger than 8-10 $\mathrm{mm}^{2}$. Therefore each single block that goes into the design need to be as small as possible. Absolute area is crucial in hearing aids as it need to fit all the analog and digital circuits. 


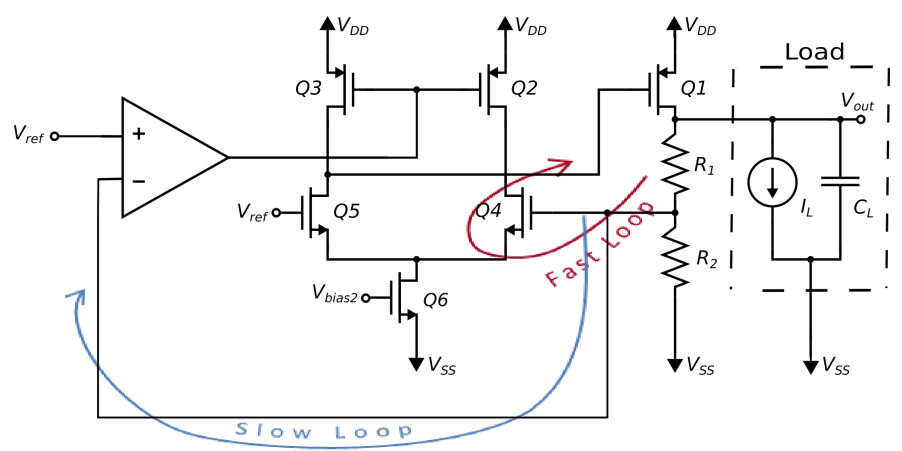

Fig. 1 Fast loop and slow loop of the proposed LDO linear voltage regulator

In this paper, Section II presents the circuit description and introduces the two regulation loops and its design details. Section III discusses the simulation results. Then, Section IV discusses the experimental results. Discussion of performance comparison with former work is presented in Section V. Finally, the conclusions of this paper are given.

This paper is an extended version of the work in [10] published in IEEE International Nordic Circuits and Systems Conference (NORCAS) in 2016.

\section{Proposed Architecture and Circuit Description}

The new design proposed in this work has two regulation loops similar to [7]. This design is a LDO using a PMOS pass transistor configured as a common source (CS) amplifier while [7] uses a NMOS as the pass transistor with a common drain stage. Refer to Fig. 1 for the circuit diagram of the proposed regulator. The design specifications target the following parameters. The regulator is supplied by nominal voltage of $1.2 \mathrm{~V}$ and outputs a voltage of 900 $\mathrm{mV}$. The load current, $I_{L}$, is $250-500 \mu \mathrm{A}$ which is stepped with a $1 \mathrm{~ns}$ rise and fall time ${ }^{1}$. The capacitance $C_{L}$ represents the load of up to $100 \mathrm{pF}$.

The fast loop consists of a differential amplifier stage, driving the common source (CS) amplifier, which include the pass transistor (Q1). The PMOS transistors in the differential stage (Q2 and Q3) are controlled by the slow loop containing the operational amplifier. The proposed design does not contain any large passive devices and has a low count of transistors. The simplicity allows for easy and area efficient implementation, while demonstrating good performance. Moreover, reaching stability is simpler compared to other designs due to the low number of poles and zeros. The following sections describe the two control loops in detail. The circuit was biased from two different current sources for debugging purposes. The full circuit diagram can be found in Fig. 2 .

1 Edge time of $1.5 \mu$ s was used for measurements and compared to simulations due to measurement limitations 


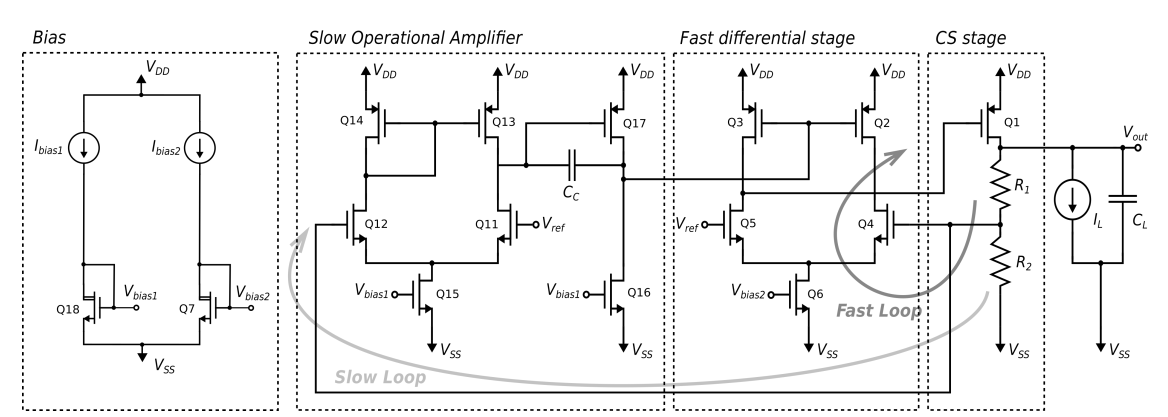

Fig. 2 Full schematic of the proposed LDO linear regulator

\subsection{Principle of Operation of the Fast Loop}

The fast loop directly regulates the gate of the pass transistor. Its purpose is to suppress the spikes in the output voltage, $V_{\text {out }}$, which is due to a step in the load. The overall performance of the regulator is impacted by the amplitude of the voltage spikes and the recovery time. By assuming the fast loop constitutes an underdamped system, the gain bandwidth product (GBWP) of the open loop gain will be inversely proportional to the settling time $T_{s}$. Therefore we will design the fast loop to have large GBWP. There is a trade-off between the circuit quiescent current in fast loop stage and the GBWP of the loop. As can be seen in Fig. 2, this loop starts at the gate of Q4 and ends at the drain of Q1.

The open loop transfer function, $A_{O L}(s)$, is described in (1). In order to analyze the loop, the equation was divided into two parts, CS stage $H_{1}(s)$ described in (2) and differential stage $H_{2}(s)$ described in (5). From the analysis of the transfer function it can be realized that the parasitic capacitance and resistance of the pass transistor (Q1) dominate the poles $\omega_{p 1}(3)$ and $\omega_{p a}(6)$. The gate-source capacitance is $C_{g s 1}$ and $C_{g d 1}$ is the gate-drain capacitance of Q1. The output resistance is represented by $r_{d s 1}$ and $g_{m 1}$ is the transconductance of Q1. The analysis was done with a load capacitance $C_{L}$ to understand its impact on the system, therefore in the zero load case $C_{t}=C_{g d 1}$. In this work the maximum value of the load capacitance was $100 \mathrm{pF}$. The transfer function $H_{1}(s)$ has two poles $\omega_{p 1}$ and $\omega_{p 2}$, both are depended on $C_{t}, C_{g d 1}$ and $C_{g s 1}$. The dominant pole $\omega_{p 1}$ denominator include also $R_{t}$ and $R_{s}$. Moreover, the second pole is proportional to the transistors transconductance, having a large $g_{m 1}$ will push $\omega_{p 2}$ to higher frequencies.

$$
\begin{gathered}
A_{O L}(s)=H_{1}(s) H_{2}(s) \frac{R_{2}}{R_{1}+R_{2}} \\
H_{1}(s)=-g_{m 1} R_{t} \frac{1}{\left(1+\frac{s}{\omega_{p 1}}\right)\left(1+\frac{s}{\omega_{p 2}}\right)} \\
\omega_{p 1} \approx \frac{1}{C_{t} R_{t}+R_{s}\left(C_{g s 1}+g_{m 1} R_{t} C_{g d 1}\right)}
\end{gathered}
$$




$$
\omega_{p 2} \approx \frac{g_{m 1} C_{g d 1}}{C_{g s 1} C_{g d 1}+C_{g s 1} C_{L}+C_{g s 1} C_{L}}
$$

For (2) - (4), $R_{t}=r_{d s 1}\left\|\left(R_{1}+R_{2}\right), C_{t}=C_{L}+C_{g d 1}, R_{s}=r_{d s 3}\right\| r_{d s 5}$. From $H_{2}(s)$ transfer function, the dominant pole $\omega_{p a}$ is set by $R_{d i f f}$ and $C_{g d 1}$. The second pole $\omega_{p b}$ and zero $\omega_{z}$ denominators are $C_{g}$ which is equal to the sum of $C_{g s 2}$ and $C_{g s 3}$ while both nominators are proportional to $g_{m 3}$. The differential stage and common source stage set the gain of the fast loop. By maximizing $g_{m 1}$ we can achieve higher gain for the CS stage. The poles and zeros were selected in the design of the fast loop to achieve high GBWP. The high W/L ratio of Q1 will introduce a large gate capacitance and transconductance which on one hand, will dominate the frequency response of the fast loop. On the other hand, a large pass transistor will also cause high parasitic capacitances which will impact the regulator performance. This big capacitance will also push the non-dominate poles to lower frequencies and potentially closer together, and therefore at some point compromise the system stability.

$$
\begin{gathered}
H_{2}(s)=-g_{m 5} R_{\text {diff }} \frac{\left(1+\frac{s}{\omega_{z}}\right)}{\left(1+\frac{s}{\omega_{p a}}\right)\left(1+\frac{s}{\omega_{p b}}\right)} \\
\omega_{p a} \approx \frac{1}{R_{\text {diff }} C_{g d 1}} \\
\omega_{p b} \approx \frac{g_{m 3}}{C_{g}} \\
\omega_{z} \approx \frac{2 g_{m 3}}{C_{g}}
\end{gathered}
$$

Where, $C_{g}=C_{g s 2}+C_{g s 3}, R_{d i f f}=r_{d s 3} \| r_{d s 5}$. The resistors $R_{1}$ and $R_{2}$ are used to set the gate voltage of $\mathrm{Q} 4$ and keep the transistor in saturation. The feedback resistance network values is $R_{1}=300 k \Omega$ and $R_{2}=600 \mathrm{k} \Omega$. The quiescent current in the differential stage of the fast loop, see Fig. 2, should be minimized to achieve overall low current consumption. By choosing the $W / L$ ratio as mentioned for Q1, the output capacitance of this stage will mainly be the gate capacitance of $\mathrm{Q} 1, C_{g 1}$, which will be larger than the capacitances of the other nodes. The gain of the differential stage in the fast loop is set primarily by the output resistance of transistors Q3 and Q5. Another aspect is the power supply rejection ratio which can be increased by using larger length for transistors Q2-Q5.

When current step loads are applied, ringing can occur on the output of the regulator due to low phase margin of the loop response. Therefore it is desirable to keep the fast loop phase margin above 75 degrees at maximum expected load capacitance. 
2.2 Principle of Operation of the Slow Loop

The role of the slow loop is to control the gate voltage of transistors Q2 and Q3 and thereby stabilize the DC level at $V_{\text {out }}$. A two stage operational amplifier (OpAmp) with Miller capacitor compensation has been utilized for this function. The slow loop is designed to consume a low quiescent current and therefore will have low power consumption. Transistors Q11 to Q18 and the miller compensation capacitor constitute the OpAmp as can be seen in Fig. 2. The slow loop starts at the gate of Q12, then through the OpAmp, proceed from the gate to the drain of Q3 and then from the gate to the drain of Q1. In order not to degrade the frequency response of the fast loop, this OpAmp has a unity gain frequency approximately two decades below that of the fast loop. Therefore the dominate pole of the OpAmp was placed at a low frequency, at $100 \mathrm{~Hz}$ as can be seen in the OpAmp frequency response in Fig. 3. We use a large Miller capacitor to move the dominant pole to lower frequency without affecting the second pole. Moreover, the loop has to be stable to maintain the stable operation of the whole system.

When the steps in $I_{L}$ occur, the OpAmp must be able to drive the gate of Q2 and Q3 without slewing the transient. Therefore, the common source stage of the OpAmp must provide a sufficiently large drain current, $I_{D 16}$. The required $I_{D 16}$ can be reduced by choosing a lower $\mathrm{W} / \mathrm{L}$ for transistors Q2 and Q3 to reduce the parasitic capacitance related to the gate. When designing the OpAmp for the slow loop there is a trade-off between the GBWP of the differential stage, the transistor dimensions of Q2 and Q3 and the necessary $I_{D 16}$ to reduce slewing.

Table 1 Device dimensions and drain current

\begin{tabular}{crrrr}
\hline Device & Width $[\mu \mathrm{m}]$ & Length $[\mu \mathrm{m}]$ & $I_{Q}[\mu \mathrm{A}]$ & $g_{m}[\mu \mathrm{A} / \mathrm{V}]$ \\
Q1 & 4000 & 0.18 & 1.0000 & 6532 \\
Q2,Q3 & 4 & 1 & 4.1280 & 43.980 \\
Q4,Q5 & 30 & 1 & 4.1280 & 112.920 \\
Q6 & 4 & 2 & 8.2560 & 89.780 \\
Q7 & 1 & 2 & 2.0000 & 22.240 \\
Q11,Q12 & 64 & 1 & 0.0157 & 0.445 \\
Q13,Q14 & 2 & 8 & 0.0157 & 0.316 \\
Q15 & 2 & 1 & 0.0315 & 0.855 \\
Q16 & 64 & 1 & 1.0210 & 27.800 \\
Q17 & 32 & 1 & 1.0210 & 23.460 \\
Q18 & 64 & 1 & 1.0210 & 27.040 \\
\hline
\end{tabular}

The design based on a slow and fast loop discussed above lead to the device dimensions, quiescent currents and transconductance presented in Table 1. The total quiescent current is $10.3 \mu \mathrm{A}$. A value of $4 \mathrm{pF}$ was chosen for $C_{C}$. 


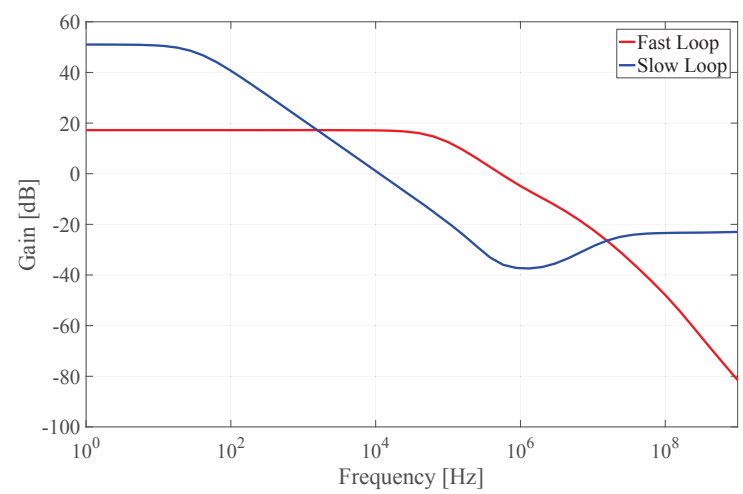

Fig. 3 Simulated open loop frequency response of the slow and fast loops, without extracted parasitics. $C_{L}=0$
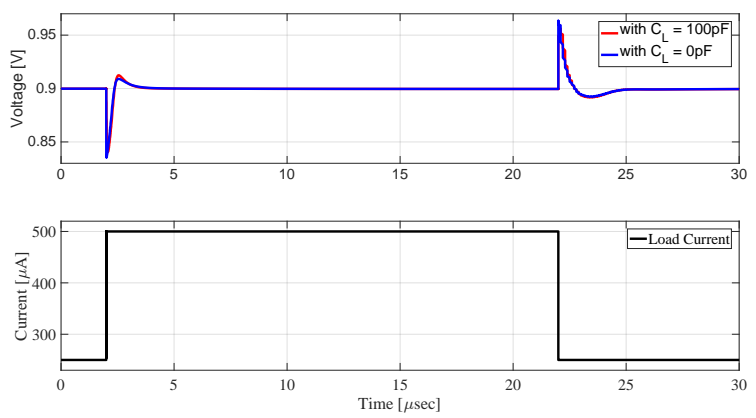

Fig. 4 Post layout transient response simulation of the complete circuit

\section{Simulation Results}

The proposed capacitor-free LDO linear voltage regulator has been implemented in a $180 \mathrm{~nm}$ CMOS process. The presented results are based on the post layout simulation. The bias current in the CS stage was $251 \mu \mathrm{A}$, when the load current is $250 \mu \mathrm{A}$. Current of $8.256 \mu \mathrm{A}$ was distributed at the differential stage and $1.05 \mu \mathrm{A}$ to the OpAmp, giving a total quiescent current consumption of $10.3 \mu \mathrm{A}$.

Post-layout simulation has been performed. Fig. 3 shows the open loop frequency response of the slow and fast loops at $C_{L}=0$. The slow loop unity gain frequency is approximately two decades below that of the fast loop as we required. The PSRR is shown in Fig. 5 , the value at $1 \mathrm{kHz}$ with and without the load capacitor is $63 \mathrm{~dB}$.

The design of intended for hearing aids, in such applications two audio channel are used, one of channels is continuously turned on and therefore a constant DC output current of $250 \mu \mathrm{A}$ is needed. When needed to power up the other channel another $250 \mu \mathrm{A}$ are required therefore the load step was set 


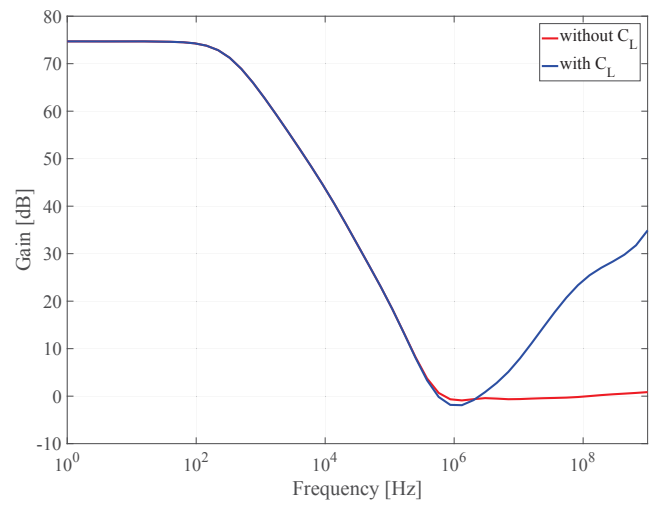

Fig. 5 Power Supply Rejection Ratio

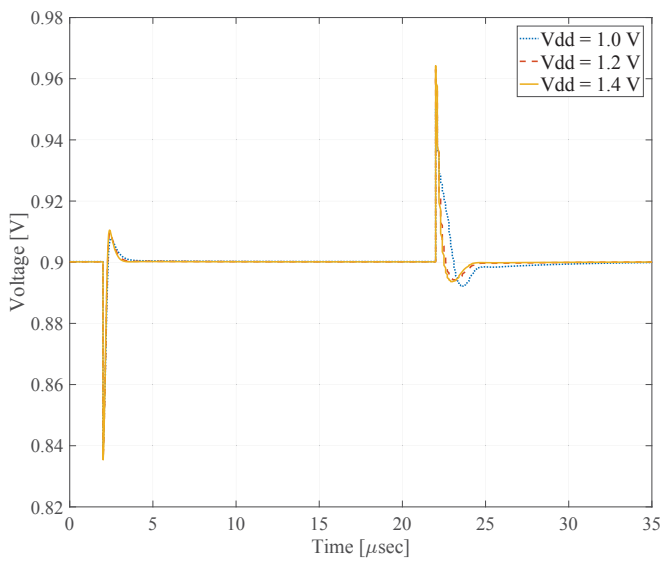

Fig. 6 Transient response simulation of the complete circuit with different supply voltages

from $250-500 \mu \mathrm{A}$. The transient response of the capacitor-free LDO voltage regulator for a current step of $250-500 \mu \mathrm{A}$ with a rise and fall time of $1 \mathrm{~ns}$ is shown in Fig. 4. The simulation was preformed with and without $C_{L} . \Delta V_{\text {out }}$ without a load capacitance is $64 \mathrm{mV}$, while for $C_{L}=100 \mathrm{pF}$ the spikes reach $56 \mathrm{mV}$. It should be noted that a smaller current step or longer rise time will decrease the spikes. Fig. 6 presents the transient analysis with different voltage supplies.

\section{Experimental Results}

The layout is presented in Fig. 7 shows the chip image with the proposed regulator layout. The LDO has been designed with measures $174 \mu \mathrm{m}$ x 68 $\mu \mathrm{m}$ which occupies an area of approximately $0.012 \mathrm{~mm}^{2}$. Common centroid matching and dummy devices have been used. The pass transistor Q1, differ- 


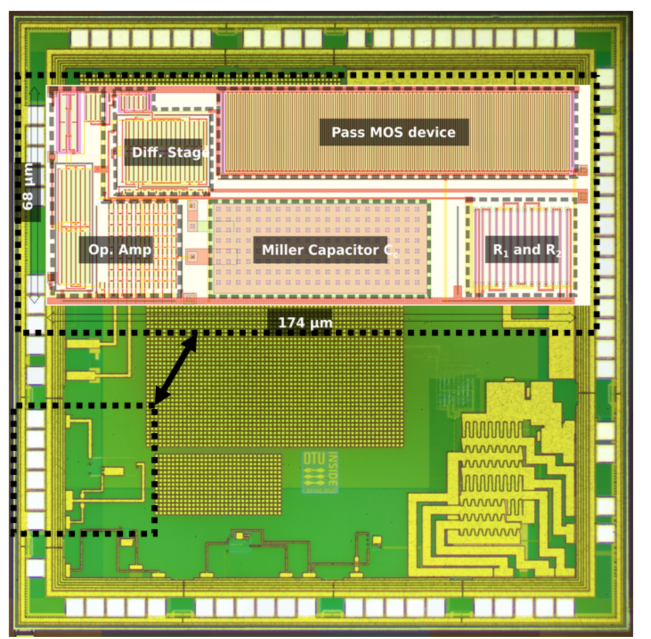

Fig. 7 Chip image with the layout of the proposed LDO linear regulator

ential stage, op-amp stage, resistors and the compensating capacitor can be seen in the layout figure. Most of the area is consumed by the power transistor and the Miller compensation capacitor.

An external reference voltage $V_{\text {ref }}$ of $0.6 \mathrm{~V}$ and resistors $R_{L 1}=3.6 \mathrm{k} \Omega$ and $R_{L 2}=3.6 \mathrm{k} \Omega$ were used to imitate the DC load current and step. This set-up can be seen in Fig. 8. To verify the operation of the proposed regulator, a load current step from $250-500 \mu \mathrm{A}$ with a rise and fall time of $1.5 \mu$ s was applied to the LDO with and without the load capacitor. The current step was implemented using the resistor $R_{L 2}$ and NMOS transistor with a signal generator connected to the gate to create the step from $0-250 \mu \mathrm{A}$. While $R_{L 1}$ is used to set the DC output current to $250 \mu \mathrm{A}$. The load step was not implemented on-chip, this caused a measurement limitation setting the current step edge time to the required 1 ns.

The transient response of the proposed design showed a maximum overshoot and undershoot of $26 \mathrm{mV}$ and $26 \mathrm{mV}$ with settling time of $3.5 \mu$ s and 1.5

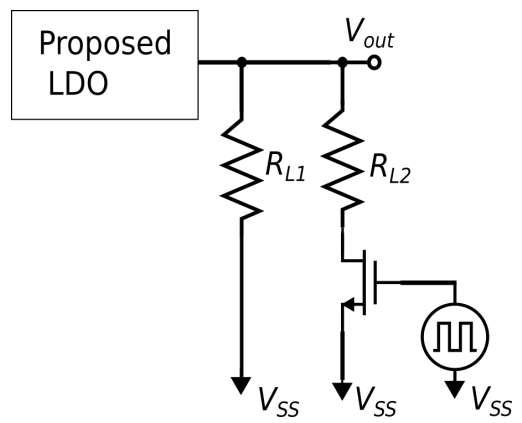

Fig. 8 Setup for LDO measurements 


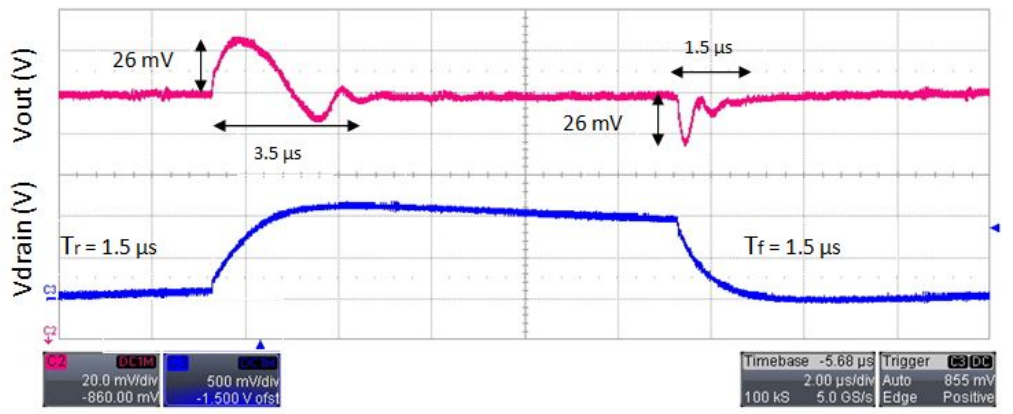

Fig. 9 Measured load transient response of the proposed LDO regulator without load capacitor
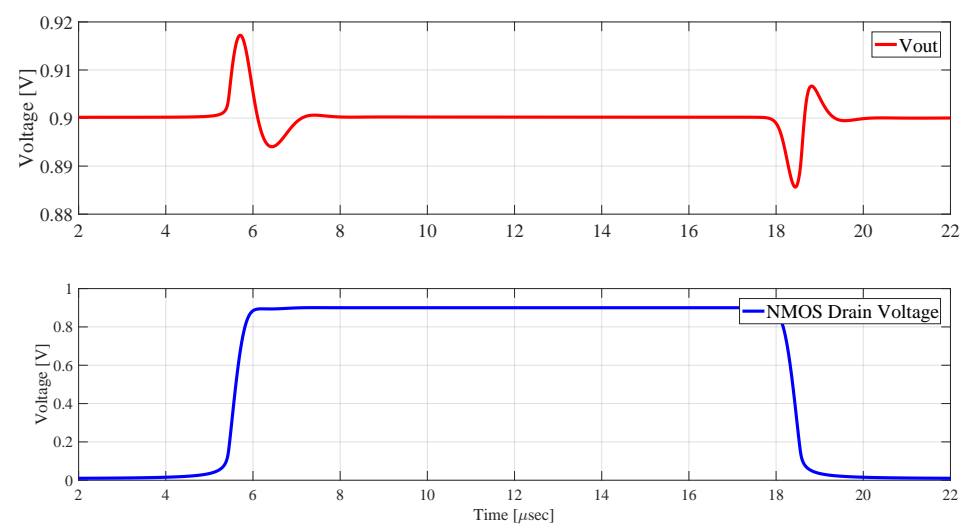

Fig. 10 Post layout transient response simulation of the proposed LDO regulator without load capacitor and with edge time $1.5 \mu \mathrm{s}$

$\mu$ s respectively, shown in Fig. 9. The transient response with a load capacitor was similar to Fig. 9 and therefore is not presented. The quiescent current is $10.5 \mu \mathrm{A}$ as expected like in the simulations. The circuit is intended to operate with an on-chip load as described in the simulation section, the edge time simulated was $1 \mathrm{~ns}$. With the measurement setup we could not produce such a steep current step. The experimental current step rise and fall time were set to $1.5 \mu$ s and compared with the simulation results with same edge time. We assume that the correlation between the simulation and measurements results with rise/fall time of $1.5 \mu \mathrm{s}$ can also be applied when the edge time is set to 1 ns.

The simulation results with rise and fall time of $1.5 \mu$ s are shown in Fig. 10. The simulation overshoot and undershoot is $18 \mathrm{mV}$ and the settling time is equal to $3 \mu \mathrm{s}$. The simulation and measurements are quite compatible but not identical due to uncompleted model like the NMOS transistor for the setup and other phenomenons. 


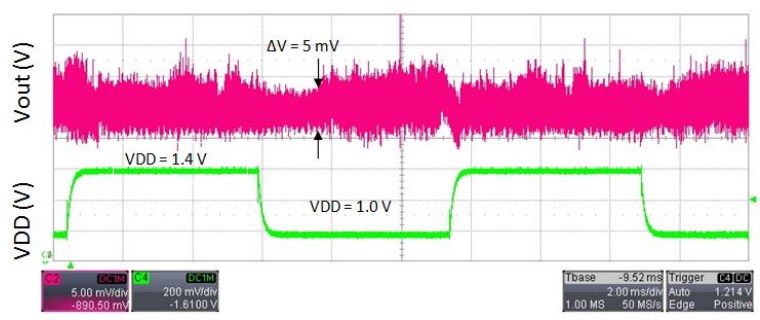

Fig. 11 Measured line transient response of the proposed LDO regulator

The measured line transient response of the proposed LDO without the use of an output capacitor is shown in Fig. 11. $V D D$ varying from $1.0 \mathrm{~V}$ to $1.4 \mathrm{~V}$, with rise and fall time $0.3 \mathrm{~ms}$. The results include noise, we can conclude that the line regulation is smaller than $\frac{5 m V}{0.4}=12.5 \mathrm{mV} / \mathrm{V}$.

\section{Performance Comparison}

The presented theory and results of the proposed LDO linear voltage regulator show that external capacitor can be replaced by the design proposed. This design is suitable to supply low current to internal circuitry like needed in hearing aids. The design is simple to implement, with small area, which makes it ideal for a system-on chip. Simulations show good performance when compared with known capacitor-free topologies. For the purpose of comparison with other regulators we define a figure of merit $(F o M)$ from [4]. This is used for standardized comparison in capacitor-free regulators as in the table. For this parameter, the smaller the $F_{O M}$, the better the transient response of the regulator.

$$
F_{O M}=K \frac{\Delta V_{O U T, p p} I_{Q}}{\Delta I_{\text {out }}}
$$

Where, $\Delta V_{O U T, p p}$ is the sum of the undershoot and overshoot and $K$ is the edge time ratio which is defined by

$$
K=\frac{\Delta t \text { used in the measurement }}{\text { smallest } \Delta t \text { among the designs for comparison }}
$$

The unit of the FoM is volt as noted in Table 2 . The $K$ factor depends on the designs considered for comparison, because the edge time of our simulation work is the smallest, it $K$ factor is equal to 1 while for the measurements results the edge time of $1.5 \mu$ s will result in $K=1500$.

The performance comparison between the proposed design and some selected published LDOs is shown in Table 2. The FoM of the proposed design when comparing to similar designs is the second lowest for the simulation results. For the measurements the FoM is quite high as a result of the large 
Table 2 Comparison of existing work

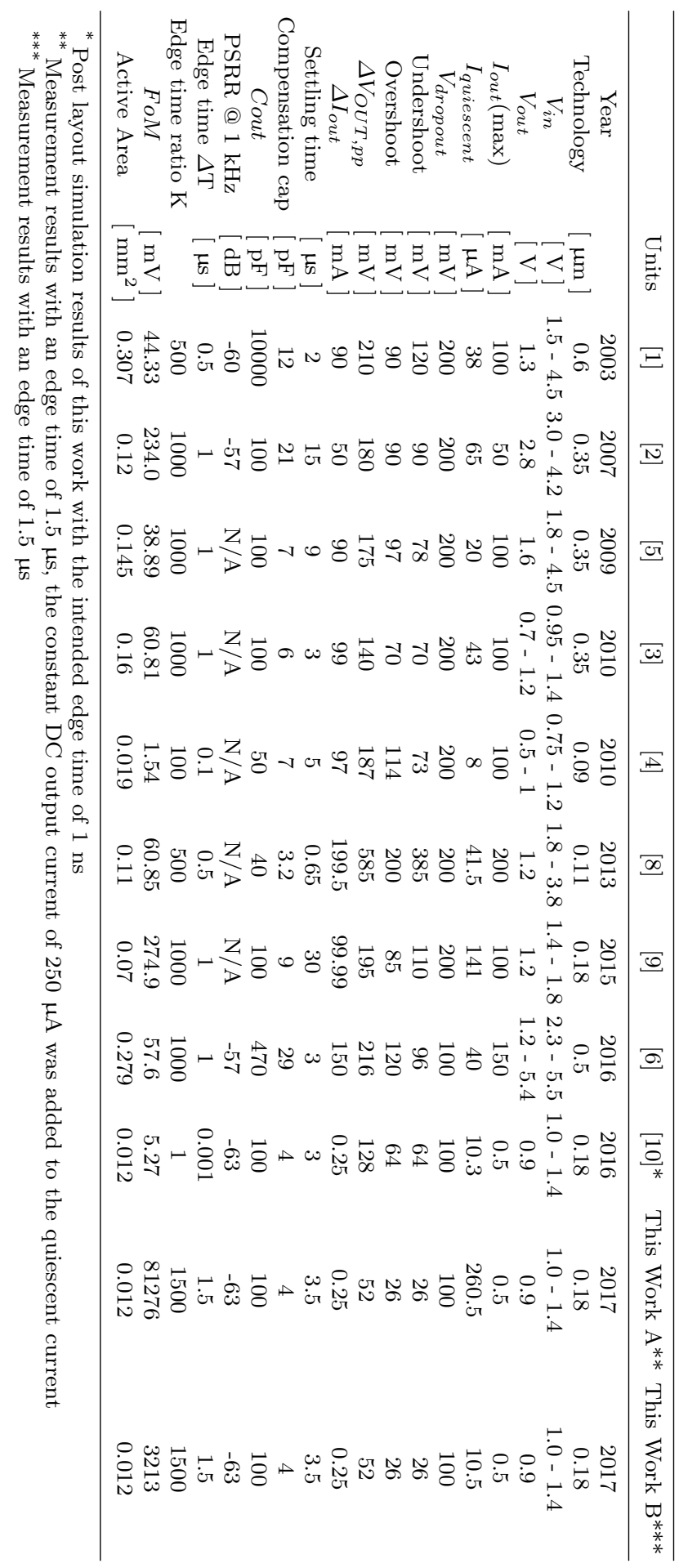


edge time from the setup limitations. Moreover, the DC output current of 250 $\mu \mathrm{A}$ (needed continuously to power one of the audio channel) decreases the FoM significantly when added to the quiescent current as can be seen in This Work A, Table 2. The column This Work B is the case when considering only the quiescent current of $10.5 \mu \mathrm{A}$ without adding the intended static load of $250 \mu \mathrm{A}$, the improvement of the FoM is by a factor of 25 . It should be noted that although the FoM is not ideal for comparing low power regulators it is still the most relevant FoM for capacitor-free LDOs, hence used in this paper. Our design has the smallest chip area, with the second lowest quiescent current of $10.5 \mu \mathrm{A}$ while the load capacitance can be as large as $100 \mathrm{pF}$. Not only does the proposed regulator consume low power, but it provides a low dropout voltage and fast settling time.

\section{Conclusion}

We have demonstrated a new capacitor-free low-dropout linear regulator for hearing aids in 180-nm CMOS technology. The structure, post layout simulation, measurements and performance comparing have been provided. The proposed LDO with 0-100 pF load capacitance is stable throughout the load range without using any decoupling capacitor. The proposed regulator has proven a good transient performance. The internal compensating capacitor is as small as $4 \mathrm{pF}$ and the chip total area is $0.012 \mathrm{~mm}^{2}$. The LDO voltage regulator can operate with supply voltage between $1.0-1.4 \mathrm{~V}$ while having a quiescent current of $10.5 \mu \mathrm{A}$ and small $\Delta V_{\text {out }}$ due to the two regulation loops. The achieved specification of the proposed LDO makes it suitable for hearing aids and similar SoC applications.

\section{References}

1. Leung, K. N., \& Mok, P. K. (2003). A capacitor-free CMOS low-dropout regulator with damping-factor-control frequency compensation. IEEE Journal of Solid-State Circuits, 38(10), 1691-1702.

2. Milliken, R. J., Silva-Martinez, J., \& Sanchez-Sinencio, E. (2007). Full on-chip CMOS low-dropout voltage regulator. IEEE Transactions on Circuits and Systems I: Regular Papers, 54(9), 1879-1890.

3. Or, P. Y., \& Leung, K. N. (2010). An output-capacitorless low-dropout regulator with direct voltage-spike detection. IEEE Journal of Solid-State Circuits, 45(2), 458-466.

4. Guo, J., \& Leung, K. N. (2010). A 6- $\mu$ W Chip-Area-Efficient Output-Capacitorless LDO in 90-nm CMOS Technology. IEEE Journal of Solid-State Circuits, 45(9), 1896-1905.

5. Ho, E. N., \& Mok, P. K. (2010). A capacitor-less CMOS active feedback low-dropout regulator with slew-rate enhancement for portable on-chip application. IEEE Transactions on Circuits and Systems II: Express Briefs, 57(2), 80-84.

6. Hong, S. W., \& Cho, G. H. (2016). High-gain wide-bandwidth capacitor-less low-dropout regulator $(\mathrm{LDO})$ for mobile applications utilizing frequency response of multiple feedback loops. IEEE Transactions on Circuits and Systems I: Regular Papers, 63(1), 46-57.

7. Deleuran, A. N., Lindbjerg, N., Pedersen, M. K., Muntal, P. L., \& Jørgensen, I. H. (2015, October). A capacitor-free, fast transient response linear voltage regulator in a 180nm CMOS. In Nordic Circuits and Systems Conference (NORCAS): NORCHIP \& International Symposium on System-on-Chip (SoC), 2015 (pp. 1-4). IEEE. 
8. Kim, Y. I., \& Lee, S. S. (2013). A capacitorless LDO regulator with fast feedback technique and low-quiescent current error amplifier. IEEE Transactions on Circuits and Systems II: Express Briefs, 60(6), 326-330.

9. Maity, A., \& Patra, A. (2016). Tradeoffs aware design procedure for an adaptively biased capacitorless low dropout regulator using nested Miller compensation. IEEE Transactions on Power Electronics, 31(1), 369-380.

10. Yosef-Hay, Y., Muntal, P. L., Larsen, D. Ø., \& Jørgensen, I. H. (2016, November). Capacitor-free, low drop-out linear regulator in a $180 \mathrm{~nm}$ CMOS for hearing aids. In Nordic Circuits and Systems Conference (NORCAS), 2016 IEEE (pp. 1-5). IEEE.

11. Ming, X., Li, Q., Zhou, Z. K., \& Zhang, B. (2012). An ultrafast adaptively biased capacitorless LDO with dynamic charging control. IEEE Transactions on Circuits and Systems II: Express Briefs, 59(1), 40-44.

12. Chen, C. M., \& Hung, C. C. (2013). Enhanced active feedback technique with dynamic compensation for low-dropout voltage regulator. Analog Integrated Circuits and Signal Processing, 75(1), 97-108.

13. Lam, Y. H., \& Ki, W. H. (2008, February). A 0.9 V 0.35 m adaptively biased CMOS LDO regulator with fast transient response. In Solid-State Circuits Conference, 2008 ISSCC 2008. Digest of Technical Papers. IEEE International (pp. 442-626). IEEE.

14. Banerjee, J., \& Nandurkar, T. S. (2005). U.S. Patent No. 6,933,772. Washington, DC: U.S. Patent and Trademark Office.

15. Jackum, T., Maderbacher, G., Pribyl, W., \& Riederer, R. (2011, May). Fast transient response capacitor-free linear voltage regulator in $65 \mathrm{~nm}$ CMOS. In Circuits and Systems (ISCAS), 2011 IEEE International Symposium on (pp. 905-908). IEEE. 